\title{
KN-15 Self-assembly of Nanoparticles Through Hydrophobic Interactions
}

Marek Grzelczak ${ }^{1,2}$

1. CIC biomaGUNE, San Sebastian, Spain

2. Ikerbasque, Basque Foundation for Science, Spain

email:mgrzelczak@cicbiomagune.es

Bottom-up self-assembly of nanoscale objects is a central premise of nanotechnology. Besides tremendous progress in last decades, the liquid-phase self-assembly of nanoparticles remains theoretically and experimentally challenging especially when dealing with non-specific interactions. We exploit hydrophobic interactions to show that polystyrene (PS)-stabilized nanoparticles dispersed in tetrahydrofuran (THF) aggregate upon addition of water to form a variety of complex architectures (Figure 1$).^{[1-6]}$ The final length-scale, interparticle distances, and shape of the aggregates are driven by nature of the surface chemistry, the geometry of particles building blocks, or rate of water addition. Since the integrity of the assemblies is maintained by non-covalent interactions, the presence of external stimuli induces disintegration, internal rearrangement or even division of the clusters that makes them an attractive material for (bio)sensing.

\section{References}

[1] M. Grzelczak, A. Sánchez-Iglesias, H. H. Mezerji, S. Bals, J. Pérez-Juste, L. M. Liz-Marzán, Nano Lett. 2012, 12, 4380.

[2] A. Sánchez-Iglesias, M. Grzelczak, T. Altantzis, B. Goris, J. Pérez-Juste, S. Bals, G. Van Tendeloo, S. H. Donaldson, B. F. Chmelka, J. N. Israelachvili, L. M. Liz-Marzán, ACS Nano 2012, 6, 11059.

[3] M. Grzelczak, A. Sánchez-Iglesias, L. M. Liz-Marzán, Soft Matter 2013, 9, 9094

[4] J. E. Galván-Moya, T. Altantzis, K. Nelissen, F. M. Peeters, M. Grzelczak, L. M. Liz-Marzán, S. Bals, G. Van Tendeloo, ACS Nano 2014, 8, 3869.

[5] M. Grzelczak, A. Sánchez-Iglesias, L. M. Liz-Marzán, CrystEngComm 2014, 16, 9425.

[6] A. L. Porta, A. Sánchez-Iglesias, T. Altantzis, S. Bals, M. Grzelczak, L. M. Liz-Marzán, Nanoscale 2015, 7, 10377.

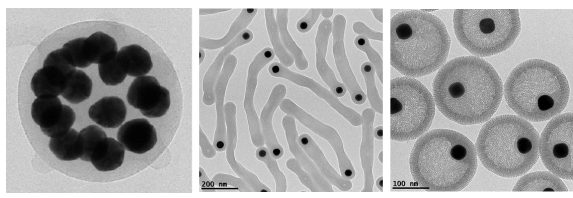

Figure 1. Solvent-induced self-assembly of polystyrene-coated gold nanoparticles. (left) Spherical clusters consisting of gold nanoparticles with $20 \mathrm{~nm}$ of diameter. The interparticle gap is controlled by the length of the polymer chains grafted on the particles surface. (middle) Eel-like polymeric structure containing metallic nanoparticles on terminus. (right) Hollow mesoporous silica capsules carrying hydrophobic nanoparticles coated with polystyrene.

Keywords: Self-assembly of nanoparticles, hydrophobic interactions 